Relations industrielles

Industrial Relations

\title{
Index et résumés de sentences arbritrales de griefs. Tome II, Montréal, Coplanam, 1985, ISBN 2-920391-03-8
}

\section{Rodrigue Blouin}

Volume 41, numéro 3, 1986

URI : https://id.erudit.org/iderudit/050243ar

DOI : https://doi.org/10.7202/050243ar

Aller au sommaire du numéro

Éditeur(s)

Département des relations industrielles de l'Université Laval

ISSN

0034-379X (imprimé)

1703-8138 (numérique)

Découvrir la revue

Citer ce compte rendu

Blouin, R. (1986). Compte rendu de [Index et résumés de sentences arbritrales de griefs. Tome II, Montréal, Coplanam, 1985, ISBN 2-920391-03-8]. Relations industrielles / Industrial Relations, 41(3), 658-659.

https://doi.org/10.7202/050243ar

Tous droits réservés @ C Département des relations industrielles de l'Université Laval, 1986
Ce document est protégé par la loi sur le droit d'auteur. L'utilisation des services d'Érudit (y compris la reproduction) est assujettie à sa politique d'utilisation que vous pouvez consulter en ligne.

https://apropos.erudit.org/fr/usagers/politique-dutilisation/ 
pears to be ready for comparable sophisticated programs for cooperative relations without resorting to those evolved in nonunion plants built on assumptions of unilateralism.

Praiseworthy are the author's efforts to assist the parties in collective bargaining in the automobile industry to deal with current challenges. But the exclusive emphasis on "environmental economic pressures» limits the usefulness of the analysis. Snide remarks on the union's reasons for introducing measures promoting employment security are not helpful. His view of unionism is narrow and confined to direct bargaining relations with employers, and therefore is inadequate for providing a base for the reconstruction of union policies and strategies. Limiting himself to the consideration of past innovations by the parties further restricts the range of innovative alternative measures. The range of subjects considered in recent bargaining sessions supports the likelihood that the parties will prove more imaginative and consider many new dimensions of their experience. As a minimum the author should review the Swedish experiences and writings and give them greater weight than what he has gleaned from the Japanese and West German writings. Nor should the author confine himself in his projections to the rigid management oriented patterns of thinking espoused by the MIT industrial relations project leaders. One hopes that he would broaden his horizons and build upon his vast investment in research and understanding of the industrial relations system in the industry to explore a wider range of constructive approaches. The results would be most helpful to the industrial relations parties ${ }^{8}$.

Solomon BARKIN

University of Massachusetts

Index et résumés de sentences arbitrales de griefs, Tome II, Coplanam, Montréal, 1985, ISBN 2-920391-03-8

Coplanam a récemment publié un nouveau recueil d'index et de résumés de sentences. Ce document comprend matériellement deux parties. On y retrouve quelques index de repérage qui orientent l'utilisateur vers les résumés apparaissant dans les divers recueils Coplanam. Ces index sont en fait une consolidation de l'ensemble des index couvrant la période 1970-1984. Le volume comprend aussi des résumés des décisions arbitrales rendues entre 1980 et 1984. Il n'est pas inutile de rappeler que Coplanam a publié en 1982, en un tome I mais en deux recueils distincts, des résumés de sentences et un jeu d'index pour la période 1970-1980. Ce tome I fut suivi, en 1983, d'un tome II comprenant en un même document des résumés de sentences arbitrales rendues entre 1980 et 1982 et un index consolidé pour la période 1970-1982. Le tome II consolide donc l'ensemble des index, mais le lecteur devra toujours manipuler les trois recueils pour ce qui est des résumés.

Les index de repérage sont toujours au nombre de trois: l'index des thèmes et sous-thèmes, l'index des arbitres et, enfin, l'index des parties. Ces index renvoient à plus de 4,000 résumés. La qualité antérieure a été maintenue et il s'agit, rappelons-le, d'index bien faits. Ils renvoient au numéro des résumés des diverses sentences, lesquelles sont présentées par ordre numérique. Cette méthode oblige à feuilleter chacun des recueils pour localiser exactement le résumé recherché. Cette façon de faire est agaçante; il aurait convenu d'indiquer la pagination.

8 For a similar approach to that offered by the reviewer see Winton HIGGINS, «Political Unionism and the Corporatist Thesis», Economic and Industrial Democracy, vol. 6, no. 3, August 1985, pp. 349-81. 
Les résumés qui apparaissent en ce tome II sont de même confection que ceux déjà publiés dans les recueils antérieurs. Il s'agit de résumés succints qui présentent l'essentiel des positions patronale et syndicale à l'arbitrage ainsi que l'essence de la décision arbitrale. Ces résumés ne sont pas toujours une source sûre pour assurer une compréhension adéquate de la portée de la décision, en ce sens que le droit régissant alors les parties ainsi que les faits ne sont pas suffisamment particularisés; il faut donc, après cette première démarche qui permet de faire une collecte rapide de la matière susceptible d'intérêt, consulter les sentences.

En définitive, le service offert par Coplanam est honnête et ne manque pas d'intérêt. Il facilite indéniablement la collecte des données. Il ne faudrait surtout pas croire que cet instrument de travail peut cependant fournir une réponse complète à un problème particulier. D'autant plus que l'arbitrage des griefs évolue depuis quelques années à une cadence effrénée et que les besoins des utilisateurs se font maintenant surtout sentir en termes d'informations au jour le jour.

Rodrigue BLOUIN

Université Laval

Canadian Labour Law - A Comprehensive Text, par G.W. Adams, Aurora, Canada Law Book Inc., 1985, 983 pp., ISBN 0-888-04-030-X

L'impressionnant ouvrage du professeur Adams, qui fut pendant près de cinq ans président du Ontario Labour Relations Board, se veut, pour reprendre les mots mêmes de la préface, un traité de droit du travail d'envergure pancanadienne ( $«$... a text on Labour Law written from an over-all Canadian perspective»). De l'exposé des différentes lois canadiennes du travail, ainsi que de la jurisprudence pertinente, devraient, compte tenu de bien des particularismes, ressortir certains traits dominants caractéristiques de tout cet ensemble: «It is my hope that this book will help identify the 'sameness' in Canadian Labour law and facilitate a greater awareness of the contribution each jurisdiction is making to the development of Canadian Labour law» (p. V). Il semblerait indiqué de dissiper d'emblée deux ambiguités de taille par rapport à la réalisation de l'objectif ainsi fixé, de même qu'en définitive, par rapport à sa présentation, à son titre: Canadian Labour Law. Même si cette démarche initiale risque de paraître injustement négative face à une oeuvre dont l'ampleur et la précision font naître assez spontanément l'éloge, elle aura à tout le moins, l'avantage d'appeler un chat, un chat. L'ouvrage bien identifié, ses qualités certaines n'en seront alors que plus manifestes.

En premier lieu, l'auteur fait le tour de l'encadrement juridique de ce qu'il est assez bien convenu d'appeler les rapports collectifs du travail. Il se confine toutefois à ceux-ci, à l'exclusion de l'étude de la situation juridique de la majorité des salariés qui se situent à l'extérieur de ce cadre. En d'autres termes, il s'agit d'un ouvrage qui traite du contenu usuel de «Labour Relations Law», mais non d'«Employment Law». À cet égard, le titre de l'ouvrage, quelque peu ancien déjà, de Carrothers (Collective Bargaining Law in Canada) était plus exact. Le vocable "droit du travail», qui rend bien «labour law», inclut, tant d'un point de vue théorique que pratique, l'un et l'autre des deux sujets précédents. Constatons de surcroît l'amplification de l'intervention législative et le renouveau en partie du droit touchant l'ensemble du salariat au cours des dernières années et de façon générale au pays (prohibition de la discrimination dans l'emploi, santé et sécurité du travail, protection à l'encontre de différents types de licenciement...).

En second lieu, malgré la présence de certains éléments pertinents, l'ouvrage, du moins dans une véritable perspective juridique scientifique, se confine au monde des provinces cana- 